\title{
Influence of a Ridge on the Low-Frequency Ground Wave
}

\author{
James R. Wait and Anabeth Murphy
}

\begin{abstract}
The problem of a plane wave incident on a semielliptical boss on an otherwise perfectly conducting flat ground plane is considered. A solution in terms of elliptic wave functions is obtained. Numerical values of the field on the near and far side of this idealized ridge are given for a base width of about two-thirds of a wavelength and various ellipticity ratios.
\end{abstract}

It is now relatively straight forward to make predictions of ground wave propagation on a smooth, homogeneous, spherical earth. ${ }^{1,2}$ Some progress has also been made recently in devising techniques to calculate the fields over an inhomogeneous and nonsmooth earth. For example, at high frequencies, the efiect of mountains or ridges obstructing the transmission path can be treated by methods of physical optics. ${ }^{3}$ Another approach, disclosed very recently, simulates the obstacle by four spherical surfaces. ${ }^{4}$ The transmission loss over each segment is then added, being an approximation valid at very high frequencies in most cases.

At low frequencies (less than $1 \mathrm{Mc}$ ) obstacles such as ridges are, no longer, large compared to the wavelength, and it is necessary to use a different approach. A suggested model to study the effect of a nonsmooth ground for low frequencies is a semielliptical boss on an otherwise flat ground plane. Assuming that the source of vertically polarized waves is at a distance from the elliptic cylinder, large compared to the wavelength, it is sufficient to consider the incident wave to be plane. Furthermore, at low frequencies the attenuation of the incident wave is negligible for a distance of several wavelengths on either side of the ridge. For this reason, the ground plane and the surface of the ridge is considered to be perfectly conducting.

The semielliptical perfectly conducting boss is shown in figure 1 . With reference to a cartesian coordinate system $(x, y, z)$ the ground plane is $y=0$ and the surface of the boss is $x^{2} / b^{2}+$ $y^{2} / a^{2}=1$, where $b$ and $a$ are the semimajor and semiminor axes of the ellipse, respectively. Elliptic-cylinder coordinates $(u, v, z)$, which are confocal with the elliptical boss, are connected to the cartesian coordinates by

$$
\left.\begin{array}{l}
x=c \cosh u \cos v \\
y=c \sinh u \sin v \\
z=z,
\end{array}\right\}
$$

where $c$, the semifocal distance, is given by

$$
c=\left(b^{2}-a^{2}\right)^{1 / 2} .
$$

The magnetic field of the incident wave has only a $z$ component and is given by

$$
H^{\text {inc. }}=H_{0} e^{i \beta x},
$$

where $H_{0}$ is the amplitude and $\beta=2 \pi /$ wavelength. Employing an addition theorem, ${ }^{5}$ this can be rewritten in terms of a particular solution of the wave equation in elliptic-cylinder coordinates as follows.

$$
H^{\text {inc. }}=H_{0}(8 \pi)^{1 / 2} \sum_{m=0}^{\infty} \frac{S e_{m}(c, v)}{N_{m}^{e}(c)} J e_{m}(c, u) e^{i m \pi / 2},
$$

${ }^{1}$ K. A. Norton, "The calculation of the ground wave field intensity over a finitely conducting spherical earth, Proc. Inst. Radio Engrs. 29, 623 (December 1951).

${ }^{2}$ H. Bremmer, Terrestial radio waves (Elsevier Publishing Co., Ltd., New York, N. Y., 1949).

${ }^{3}$ F. H. Dickson, J. J. Egli, J. W. Herbstreit, and G. S. Wickizer, Large reductions of V. H. F. transmission loss and fading by the presence of a mountain obstacle in beyond line of sight paths, Proc. Inst. Radio Engrs. 41, 967 (August 1953). See also a discussion of this paper by J. H. Crysdale, Proc. Inst. Radio Engrs. 43, 627 (May 1955).

${ }^{4}$ K. A. Norton, P. L. Rice, and L. E. Vogler, The use of angular distance in estimating transmission loss, Proc. Inst. Radio Engrs. 43, 1488 (October 1955).

${ }^{5}$ P. M. Morse and H. Feshbach, Methods of theoretical physics 2, 1572 (McGraw Hill Book Co., New York, N. Y., 1953). 


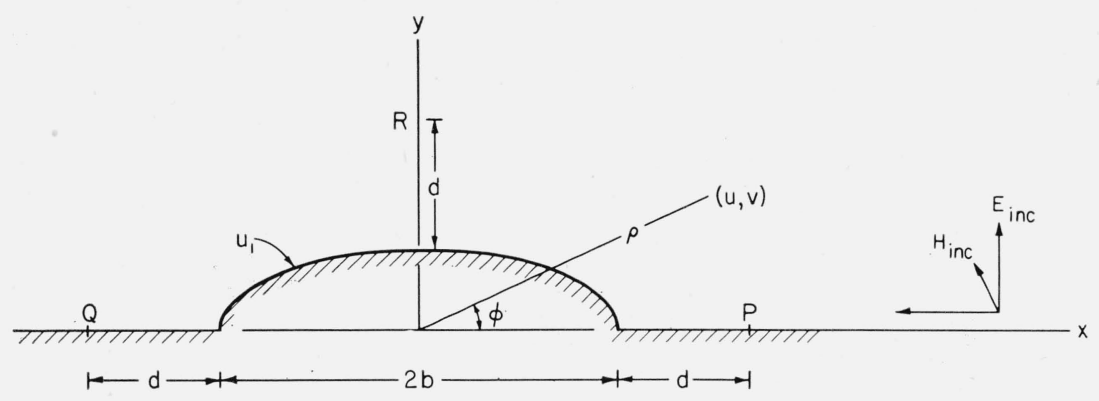

Figure 1. Semielliptical boss.

where $S e_{m}$ is the even angular Mathieu function, $J e_{m}$ is the even radial Mathieu function of the first type, and $N_{m}^{e}$ is the normalization constant for Mathieu functions of order $m$.

The secondary or scattered field, $H^{\text {sec., }}$, is now chosen to satisfy the boundary condition that the normal derivative of the total field is zero at $u=u_{1}$, the surface of the elliptic cylinder, and at $v=0$ and $\pi$, the surface of the ground plane. Furthermore, the secondary field must contain the radial Mathieu function $H e_{m}^{(2)}$ of the fourth type to comply with the radiation condition at infinity. It then follows that

$$
H^{\mathrm{sec} .}=-H_{0}(8 \pi)^{1 / 2} \sum_{m=0}^{\infty} \frac{S e_{m}(c, v)}{N_{m}^{e}(c)}\left[\frac{\frac{\partial J e_{m}(c, u)}{\partial u}}{\frac{\partial H e_{m}^{(2)}(c, u)}{\partial u}}\right]_{u=u_{1}} \quad H e_{m}^{(2)}(c, u) e^{i m \pi / 2} .
$$

The Mathieu functions are not tabulated directly but must be calculated from series expansions whose coefficients are available in tabular form. ${ }^{6}$ In the present notation

$$
S e_{2 r+p}(c, v)=\sum_{k=0}^{\infty} D e_{2 k+p} \cos [(2 k+p) v]
$$

and

$$
J e_{2 r+p}(c, u)=\left(\frac{\pi}{2}\right)^{1 / 2}(-1)^{r} \sum_{k=0}^{\infty}(-1)^{k} D e_{2 k+p} J_{2 k+p}(c \cosh u),
$$

where $r$ is an integer, $p=0$ or $1, J_{2 k+p}$ is the Bessel function of the first type or order $2 k+p$, and $D e_{2 k+p}$ are coefficients that are functions of $2 r+p$ and $c$. An expansion for $H e_{m}^{(2)}$ is obtained by replacing $J_{2 k+p}$ on the right-hand side by the Hankel function $H_{2 k+p}^{(2)}$ of the second type. As it turns out, however, this latter expansion is very poorly convergent for the present problem, and it is better to utilize the relation

$$
H e_{m}^{(2)}(u, v)=J e_{m}(u, v)-i N e_{m}(u, v),
$$

along with the expansion for the radial Mathieu of the third type, given by (see footnote 6)

and

$$
N e_{2 r}(c, u)=(-1)^{r}\left(\frac{\pi}{2}\right)^{1 / 2} \sum_{k=0}^{\infty} \frac{(-1)^{k} D e_{2 k} Y_{k}(\alpha) J_{k}(\beta)}{D e_{0}}
$$

$$
N e_{2 r+1}(c, u)=(-1)^{r}\left(\frac{\pi}{2}\right)^{1 / 2} \sum_{k=0}^{\infty}(-1)^{k} \frac{D e_{2 k+1}}{D e_{1}}\left[Y_{k+1}(\alpha) J_{k}(\beta)+Y_{k}(\alpha) J_{k+1}(\beta)\right],
$$

where $\alpha=c e^{u} / 2$ and $\beta=c e^{-u} / 2$. The functions $J_{k}$ and $Y_{k}$ are Bessel functions of the first and second kind, respectively, of order $k$. The corresponding expansions for the derivatives of the

\footnotetext{
${ }^{6}$ Tables relating to Mathieu functions (Columbia University Press, New York, N. Y., 1951).
} 


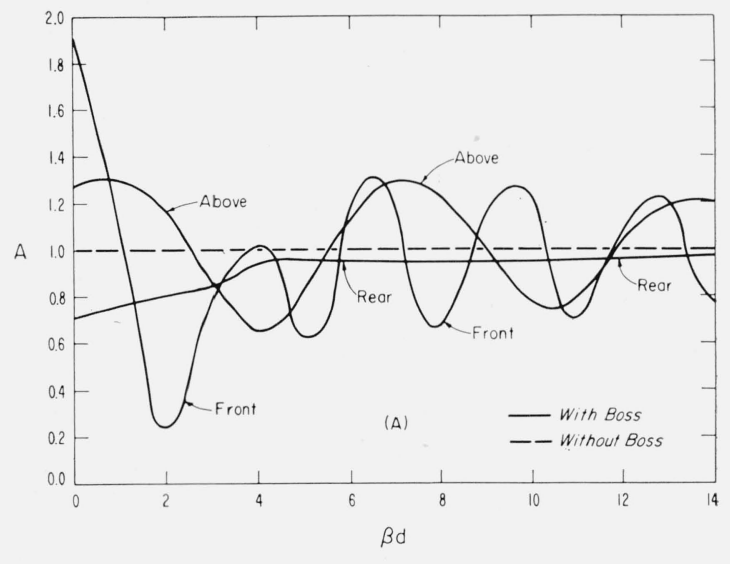

FIGURE 2 .

A, Amplitude for semicylindrical boss. $\mathrm{B}$, Phase for semicylindrical boss. $(\boldsymbol{\beta} b=2)$

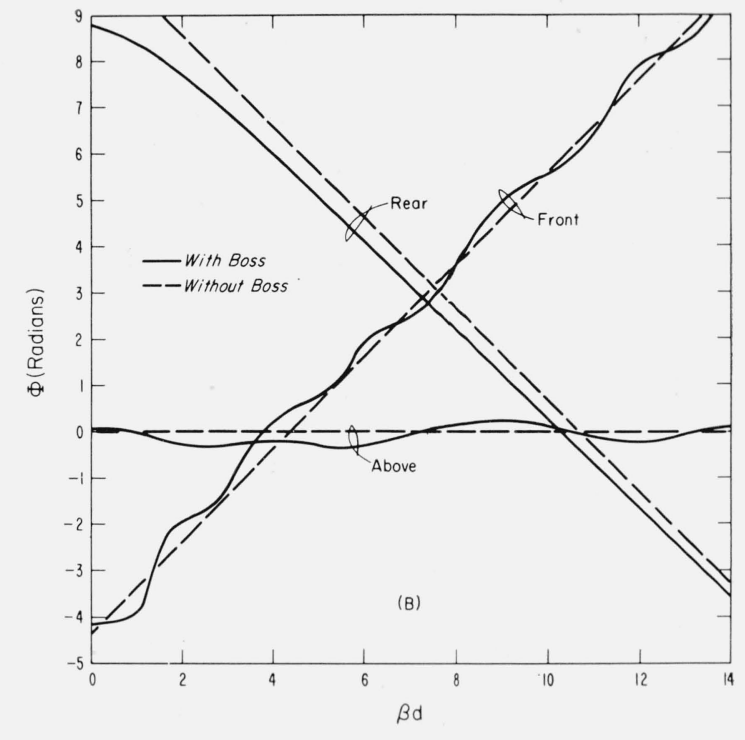

radial function are obtained in a straightforward way by differentiating with respect to $u$ and then making use of various recurrence relations for Bessel functions. Therefore,

$$
\left[\frac{\partial J e_{2 r+p}(c, u)}{\partial u}\right]_{u-u_{1}}=\left(\frac{\pi}{2}\right)^{1 / 2}(-1)^{r} \sum_{k=0}^{\infty}(-1)^{k} D e_{2 k+p}\left[\tanh u_{1}(2 k+p) J_{2 k+p}-c \sinh u_{1} J_{2 k+p+1}\right],
$$

where the argument of the Bessel functions is $c \cosh u_{1}$. Correspondingly,

$$
\left[\frac{\partial H e_{m}^{(2)}(c, u)}{\partial u}\right]_{u=u_{1}}=\left[\frac{\partial J e_{m}(c, u)}{\partial u}\right]_{u=u_{1}}-i\left[\frac{\partial N e_{m}(c, u)}{\partial u}\right]_{u=u_{1}}
$$

where

$$
\left[\frac{\partial N e_{2 r}(c, u)}{\partial u}\right]_{u=u_{1}}=(-1)^{r}\left(\frac{\pi}{2}\right)^{1 / 2} \sum_{k=0}^{\infty}(-1)^{k} \frac{D e_{2 k}}{D e_{0}}\left[Y_{k}^{\prime}(\alpha) J_{k}(\beta) \alpha-Y_{k}(\alpha) J_{k}^{\prime}(\beta) \beta\right]
$$

and

$$
\begin{aligned}
& {\left[\frac{\partial N e_{2 r+1}(c, u)}{\partial u}\right]_{u=u_{1}}} \\
& =(-1)^{r}\left(\frac{\pi}{2}\right)^{1 / 2} \sum_{k=0}^{\infty}(-1)^{k} \frac{D e_{2 k+1}}{D e_{1}}\left(Y_{k+1}^{\prime}(\alpha) J_{k}(\beta) \alpha+Y_{k}^{\prime}(\alpha) J_{k+1}(\beta) \alpha\right. \\
& \left.-Y_{k+1}(\alpha) J_{k}^{\prime}(\beta) \beta-Y_{k}(\alpha) J_{k+1}^{\prime}(\beta) \beta\right],
\end{aligned}
$$

where the prime over the Bessel functions indicates a derivative with respect to its argument.

Using the above formulas, the amplitude and phase of the field is computed for several cases. The results are normalized so that

$$
A e^{i \Phi}=\left(H^{\text {inc. }}+H^{\text {sec }}\right) / H_{0},
$$

where $A$ and $\Phi$ are the amplitude and phase of the total field relative to the incident field at $x=0$.

In figure 2 the functions $A$ and $\Phi$ are shown plotted along the ground plane in front of the ridge at $\mathrm{P}$ (i. e., $x>b, y=0$ ), above the obstacle at $\mathrm{Q}$ (i. e., $x=0, y>a$ ), and to the rear of the obstacle at $\mathrm{R}$ (i. e., $x<-b, y=0$ ). - In each case, the quantity $d$ indicates the distance measured from the elliptical suiface to the points $\mathrm{P}, \mathrm{Q}$, and $\mathrm{R}$, as illustrated in figure 1 . The curves in figure 2 refer to the case where $\beta b=\beta a=2$, so the elliptical boss has degenerated to a 

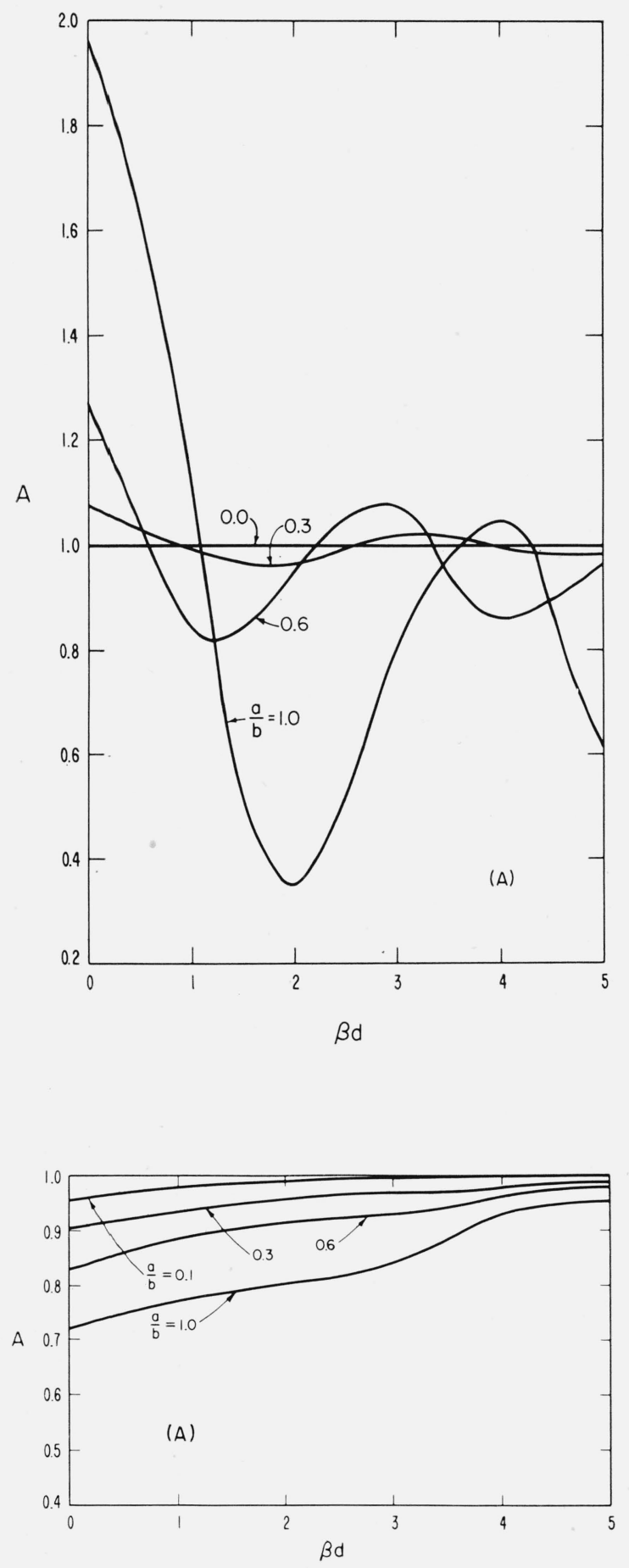

Figure 4.

A, Amplitude of field to rear of semielliptical boss. $\mathrm{B}$, Phase of field to rear of semielliptical boss.

$$
\left.\left(\boldsymbol{\beta}^{\prime}\right)=2\right)
$$

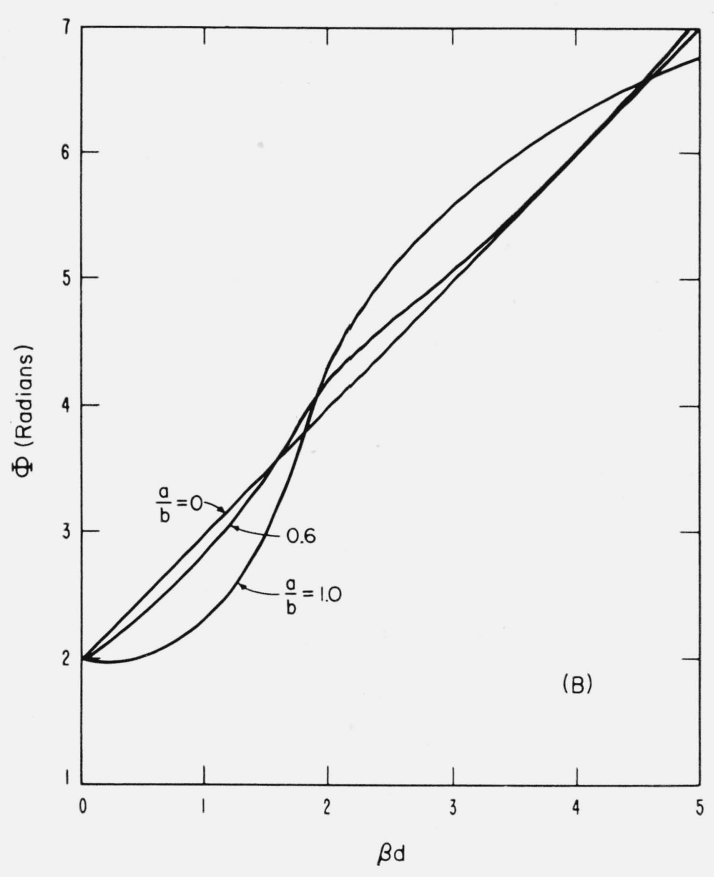

Figure 3.

A, Amplitude of field in front of semielliptical boss. $\mathrm{B}$, Phase of field in front of semielliptical boss. $(\beta b=2)$

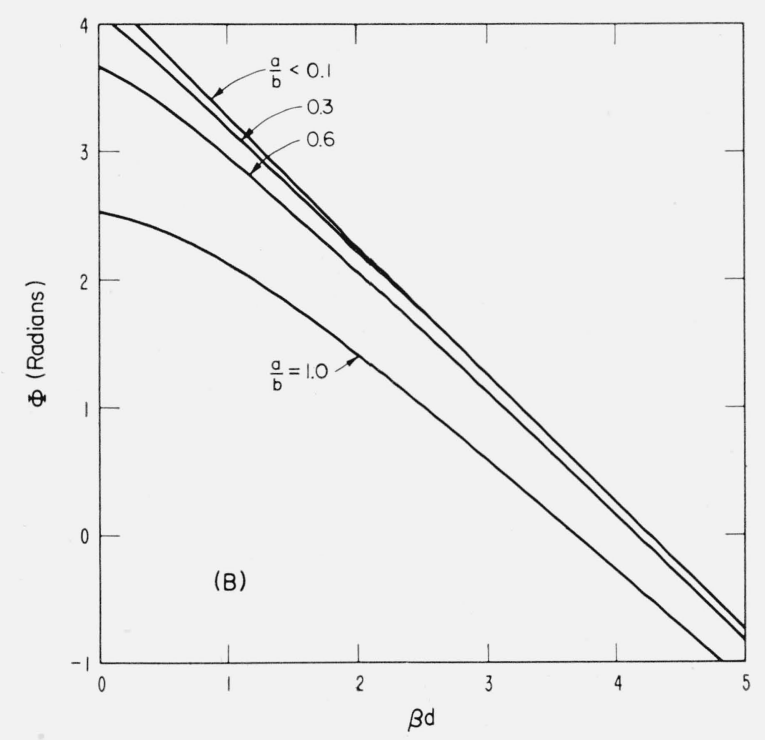


circular boss whose height is $1 / \pi$ wavelengths. The broken curves in both figures $2, \mathrm{~A}$, and $2, \mathrm{~B}$, correspond to the trivial situation where the boss is absent.

It is interesting to note in figure 2 that behind the obstacle, the amplitude of the field is reduced in amplitude by about 30 percent and gradually approaches the unobstructed value as the observer proceeds away from obstacle. The obstacle imparts an additional phase lag of the order of $60^{\circ}$ directly behind the obstacle and this value diminishes to about $15^{\circ}$ at two wavelengths behind.

In front of the obstacle, the amplitude and phase of the field oscillates about the unobstructed value as the observer proceeds toward the source as a result of the interaction of the incoming plane wave with the reflected wave. At a distance greater than a wavelength or so, the amplitude varies in the manner of a damped sinusoid with a period of one-half wavelength and a magnitude varying inversely as the square root of the distance. In the proximity of the obstacle, the structure of the field is quite complex and no simple physical interpretation seems possible. It is interesting to note, however, just in front of the obstacle (i. e., $x=b, y=0$ ) the value of the field is almost twice the value of the incident field. This would be expected on the basis of geometrical optics, which predicts a value of exactly two.

Directly above the obstacle, the field oscillates about the unobstructed value with a period approximately equal to a wavelength. The amplitude of the oscillations decreases approximately as the inverse square root of the distance.

To illustrate the effect of changing the cross-sectional shape from circular to elliptical, values of $A$ and $\Phi$ are plotted as of function $d$ both in front (fig. 3) and in the rear (fig. 4) of the elliptical boss. Various values of the ellipticity $a / b$ are shown. Of course, when $a / b$ approaches zero the field values approach the unobstructed values. It is interesting to note that the qualitative features of the curves are similar, although there is a strong dependence of the amplitude and the phase on the ellipticity ratio. In all the calculations, the width of the base of the boss (i. e., $2 b)$ has been fixed such that $\beta b=2$ or $2 b$ is $(2 / \pi)$ of a wavelength.

The extension of these results to oblique incidence is simple. For example, if the angle of the wave front makes an angle $\theta$ with the axis of the elliptical boss, the preceding results are applicable if $\beta$ is replaced by $\beta \sin \theta$.

Boulder, Colo., August 23, 1956. 